\title{
The importance of semantic similarity to the irrelevant speech effect
}

\author{
CRAIG B. NEELY and DENNY C. LECOMPTE \\ Louisiana State University, Baton Rouge, Louisiana
}

\begin{abstract}
Irrelevant speech disrupts immediate recall of a short sequence of items. Salamé and Baddeley (1982) found a very small and nonsignificant increase in the irrelevant speech effect when the speech comprised items semantically identical to the to-be-remembered items, leading subsequent researchers to conclude that semantic similarity plays no role in the irrelevant speech effect. Experiment 1 showed that strong free associates of the to-be-remembered items disrupted serial recall to a greater extent than words that were dissimilar to the to-be-remembered items. Experiment 2 showed that this same pattern of disruption in a free recall task. Theoretical implications of these findings are discussed.
\end{abstract}

A robust finding in immediate memory is that, despite explicit instructions to the contrary, background speech significantly disrupts short-term memory (e.g., Colle \& Welsh, 1976; Jones, Madden, \& Miles, 1992; LeCompte, 1994, 1995, 1996; Salamé \& Baddeley, 1982). A typical irrelevant speech effect experiment could be described as follows: Participants are presented with a list of seven to nine items on a computer screen at a rate of one or two items per second. During the visual presentation, participants hear either nothing or spoken words. After the presentation of the visual items, participants are asked to recall these items in serial order- that is, in the order they were presented. The typical finding is that recall of the visual items is significantly worse with the speech background than with the quiet background. Note, however, that although the irrelevant speech effect is usually demonstrated with visual stimuli, it is also found with auditory stimuli (see, e.g., LeCompte, 1996), and although irrelevant speech is the usual disruptive agent, tones have also been shown to disrupt memory (see, e.g., Jones \& Macken, 1993), although to a lesser extent than speech (LeCompte, Neely, \& Wilson, 1997).

Perhaps the most basic question about the irrelevant speech effect concerns the mechanism by which irrelevant speech interferes with short-term memory. Two theories have been proposed to explain this interference. Each theory offers a different mechanism for the interference.

The first theory, the phonological store hypothesis of Salamé and Baddeley $(1982,1989)$, proposes a store that holds information in a strictly phonological form. According to the hypothesis, speech has obligatory access to the phonological store. Visual information also enters

We thank Brian Bornstein, Tim Curran, Janet McDonald, and Anjali Thapar for their comments on earlier drafts of this article. Portions of this research were supported by National Institute of Mental Health Grant MH35873 to Michael J. Watkins. Correspondence should be addressed to C. B. Neely, Department of Psychology, Louisiana State University, Baton Rouge, LA 70803-5501 (e-mail: craig.neely@)compaq. com). this store, but it must be coded phonologically through an active process of subvocal rehearsal. While these two types of information are together in the phonological store, the auditory information interferes with the visual information, thereby degrading recall of the visual information. The degree of interference is a function of the phonological similarity between the background speech and the visual information.

The second theory, the changing state theory of Jones and his colleagues (e.g., Jones, 1993, 1995; Jones, Beaman, \& Macken, 1996; Jones \& Macken, 1993; Jones et al., 1992; Macken \& Jones, 1995), states that auditory and visual stimuli are processed by the individual and stored on a metaphorical blackboard as objects. Visual objects are formed via conscious serial rehearsal; this rehearsal also creates pointers between visual objects that indicate their order of presentation. Auditory objects are formed automatically, without conscious control. Different auditory objects are formed whenever a change in state is perceived. Although change in state has never been defined clearly (see LeCompte, 1996), it might be considered a perceptual change in a stimulus. Examples of speech that changes in state would include a series of different syllables (Jones et al., 1992), repetitions of the same syllable presented in a series of different spatial locations (Jones \& Macken 1995a), and repetitions of the same syllable presented in a series of different voices (Jones \& Macken, 1993). An example of speech that does not change in state would include repetitions of the same syllable in the same voice at an even rate (LeCompte, 1995). Changes in state cause pointers between auditory objects to form automatically, thereby specifying their order of presentation.

The disruption of visual short-term memory has been explained in two ways by the hypothesis. First, it has been stated (Jones et al., 1992) that during recall, an individual uses the links to reconstruct the visually presented list, but when background speech is present, the pointers from the auditory stream are confused with the pointers from the visual stream. The result of this confusion is a disruption of memory for the serial order of the visual 
stimuli. Jones (1995) also stated that changing state auditory stimuli disrupt the serial rehearsal of the visual (to-be-remembered) stimuli, thereby preventing them from forming as objects on the blackboard in the first place. Regardless of the particular explanation, the changing state hypothesis cites changes in state as the source of interference in the irrelevant speech effect rather than phonological similarity.

Both of these theories propose that the interference caused by irrelevant speech is due exclusively to perceptual characteristics of speech. The phonological store theory relies on phonology, and the changing state theory relies on even more fundamental perceptual features such as pitch or spatial location. The reliance by both theories on perceptual characteristics allows them to account for one of the more curious properties of the irrelevant speech effect - that is, that the meaning of the background speech seems unrelated to the magnitude of its disruptive effect.

A few studies have compared speech that was meaningless to the experimental participants with speech that was meaningful to the participants (Jones et al., 1992; LeCompte et al., 1997; LeCompte \& Shaibe, 1997) and failed to find any difference; however, none of these studies actually examined whether the semantic relationship between the background speech and the to-beremembered visual stimuli was important. Only three sets of experiments have examined that issue. Salamé and Baddeley's (1982) Experiment 5 manipulated semantic similarity in that way. Their experiment therefore provides some of the strongest evidence against an influence of semantic similarity in the irrelevant speech effect. Using digits as visual stimuli, they compared three kinds of background speech: digits (e.g., one, two, three), words that rhymed with digits (e.g., tun, gnu, tee), or words that were phonologically and semantically dissimilar to the digits (e.g., tennis, jelly, tipple). Although a close examination of Salamé and Baddeley's (1982) data suggests a very small increase in disruption for the digits, no significant difference was found between the digits background and the rhyming background. This failure to reject the null hypothesis of no difference may, however, be attributable to the fact that only 20 participants were included in the experiment, giving this experiment fairly low statistical power.

Bridges and Jones (1996, Experiment 4) recently replicated Salamé and Baddeley's (1982) experiment and also failed to find any difference between irrelevant speech comprising digits and irrelevant speech comprising nondigit rhyming words. If the semantic similarity between the background speech and the to-be-remembered visual items were important, the digits should have caused a larger effect than the rhymes. Again, low statistical power is a concern for this study because Bridges and Jones failed to reject the null hypothesis, yet they tested only 18 participants.

Buchner, Irmen, and Erdfelder (1996) conceptually replicated Experiment 5 of Salamé and Baddeley (1982).
Buchner et al. used two-digit numbers as the visual items and two-digit numbers, as well as phonologically matched nonwords, as irrelevant speech. They found no difference in the disruption of the visual to-be-remembered items between the background speech conditions. Also, in their second experiment, two-digit numbers were again used as the visual to-be-remembered items. The irrelevant speech comprised either the same numbers as the visual to-be-remembered items or numbers that differed by $2,5,20$, or 50 . Buchner et al. found no differences among the backgrounds. These results led them to conclude that semantic similarity has no effect on the irrelevant speech paradigm. Unlike the experimenters in the aforementioned studies, Buchner et al. used considerably more participants in each of their experiments - -78 in Experiment 1 and 96 in Experiment 2, making their null effects considerably more believable.

\section{EXPERIMENT 1}

Although the existing evidence seems to provide strong evidence that semantic similarity is unimportant to the irrelevant speech effect, there are reasons to doubt this conclusion. First, as mentioned earlier, two of the experiments comparing related and unrelated irrelevant speech included a fairly low number of participants, which suggests that statistical power may be too low to warrant rejecting the null hypothesis.

Another possible weakness of the methodology used in all of the aforementioned experiments concerns the to-be-remembered stimuli. Participants were required to remember short series of digits, and digit and nondigit irrelevant speech were compared. The problem with digits is that in the context of a serial recall experiment, they are essentially meaningless tokens. For instance, one could accomplish the task just as well without even knowing the canonical order of the digits.

Experiment 1 tried to remedy the weaknesses of earlier studies by greatly increasing the number of participants and by using more meaningful words as to-be-remembered stimuli. This first experiment was conducted before Jones and Macken (1995b), Bridges and Jones (1996), or LeCompte and Shaibe (1997) reported that phonological similarity between the irrelevant speech and the to-beremembered stimuli has no effect; therefore, it was designed to minimize the phonological similarity between the irrelevant speech and the to-be-remembered stimuli. Thus, we compared background speech comprising words known to be strong associates of the to-be-remembered stimuli with background speech comprising words that were relatively unrelated to the to-be-remembered stimuli. Thus, no irrelevant speech word was ever a to-beremembered word. Pairs of stimuli (e.g., black-white) were chosen on the basis of their strength of association in free association norms (Bilodeau \& Howell, 1965). Only the strongest associates in the association norms were used. If semantic similarity influences the magnitude of the irrelevant speech effect, the strongly associated background 
should impair recall to a greater extent than the unrelated background.

\section{Method \\ Participants. The participants were 88 students enrolled in psy- chology courses at Louisiana State University who volunteered in exchange for extra credit given in these courses.}

Materials and Design. Participants saw 63 lists of words. Each list comprised a random permutation of the words cold, dark, hard, head, man, slow, black, and king. Each list was generated separately for each participant.

The lists were presented visually in the center of an Apple Macintosh Classic II computer screen. The letters were in lowercase 48point Geneva font and were black on a white background. The tobe-remembered words were printed on a cover placed over the number keys at the top of the keypad. The words were randomly assigned to keys; however, the order of these words was the same for all participants. Participants recalled the visual words by pressing the key that corresponded to the word.

Each list was accompanied by one of three backgrounds. In the quiet condition, only ambient noise attenuated by the headphones was heard during list presentation. In the associated condition, random permutations of the words hot, light, soft, foot, woman, fast, white, and queen were heard during list presentation. These words and the to-be-remembered words listed earlier were chosen because they were the eight strongest associates in a list of free association norms (Bilodeau \& Howell, 1965). In the unrelated condition, the words frame, hill, mild, pile, scale, toy, win, and curl were heard during list presentation. These words were chosen because none of them was listed as an associate of any of the to-be-remembered words or of the words in the associated background. The words were digitized in a male voice for computer presentation and were all between 400 and $725 \mathrm{msec}$ in length. The order of the words in the background condition was determined randomly for each list and separately for each participant; however, an important constraint was that a to-be-remembered word and its associate were never presented at the same time.

Irrelevant speech backgrounds were varied within participants. The 63 lists were divided into 21 blocks of three lists each. Each block included all three background conditions in a random order that was determined separately for each block and for each participant. The participants were told to treat the first three lists as practice trials; these lists were not scored.

Procedure. Each participant sat at a computer and wore headphones attached to that computer. The instructions were presented on the computer screen and familiarized the participants with the nature of the task. The participants saw a list of words one at a time, and following each list, they tried to recall these words in the order they appeared. Each word was presented for $750 \mathrm{msec}$ and was followed immediately by the next item. Recall was accomplished by pressing a computer key that corresponded to the appropriate word Participants pressed a key labeled "blank" in positions for words they could not recall. The participants were not allowed to backspace. After the participant entered eight responses, the screen cleared, and the next list was presented after a $2-\sec$ delay.

During the presentation of some of the lists, participants heard speech through their headphones. The onset of each word in the background was synchronized with the onset of each to-beremembered word. No background speech was played during recall. The instructions at the beginning of the experiment made it clear that the participants were to ignore this speech.

\section{Results}

The participants' recall of the visually presented words was scored correct only if the word was in its correct se- rial position. Probability of recall is presented as a function of both background condition and serial position in Figure 1. The overall probability of recall was .47 for quiet, .38 for the unrelated background, and .36 for the associated background, yielding a significant main effect of background condition $\left[F(2,174)=84.01, M S_{\mathrm{e}}=0.03\right.$, $p<.001]$. Performance in the quiet condition exceeded performance in both the unrelated $[t(87)=10.03, p<$ $.001]$ and related $[t(87)=12.12, p<.001]$ speech conditions to a reliable extent. Of greater importance is the finding that the related speech condition had a significantly lower probability of recall than did the unrelated speech condition $[t(87)=3.16, p=.002]$, thereby showing that increasing the degree of semantic similarity between the irrelevant speech and the to-be-remembered visual items increased the magnitude of the irrelevant speech effect.

Although not of primary interest, there was also a significant effect of serial position $[F(14,1218)=125.47$, $\left.M S_{\mathrm{e}}=.06, p<.001\right]$, resulting from the hook-shaped curve typical of serial recall of visual stimuli (see LeCompte, 1995). There was also a significant interaction between background condition and serial position $\left[F(14,1218)=14.53, M S_{\mathrm{e}}=.01, p<.001\right]$. This interaction seems to have arisen from the fact that both the basic irrelevant speech effect and the small semantic similarity effect are more pronounced at the beginning of the list than at the end of the list. This interaction does not change the fundamental conclusion from this experiment: Semantic similarity slightly increases the magnitude of the irrelevant speech effect.

\section{EXPERIMENT 2}

Experiment 1 showed that strong associations between the to-be-remembered visual items and the irrelevant speech items can lead to an increased irrelevant speech effect. This effect was quite small, however. Why is it that the effect of semantic similarity was so small? Furthermore, why was the effect of semantic similarity virtually nonexistent in previous studies (Bridges \& Jones, 1996; Buchner et al., 1996; Salamé \& Baddeley, 1982)? One possibility is that although semantic meaning may sometimes play a role in working memory, its contribution, relative to phonology, is fairly minor.

Another, although not mutually exclusive, explanation is that serial recall of a small set of stimuli-even when these stimuli are meaningful words and not digits-does not require much semantic processing. Serial recall can be accomplished largely on the basis of phonological memory for the stimuli (see Baddeley, 1986); consequently, it may be more difficult for the semantic content of irrelevant speech to interfere with the primarily phonological processing used for serial recall of a small set of stimuli.

With this reasoning in mind, we sought to replicate the effect of semantic similarity using a task that might be more likely to involve semantic processing. Specifically, 


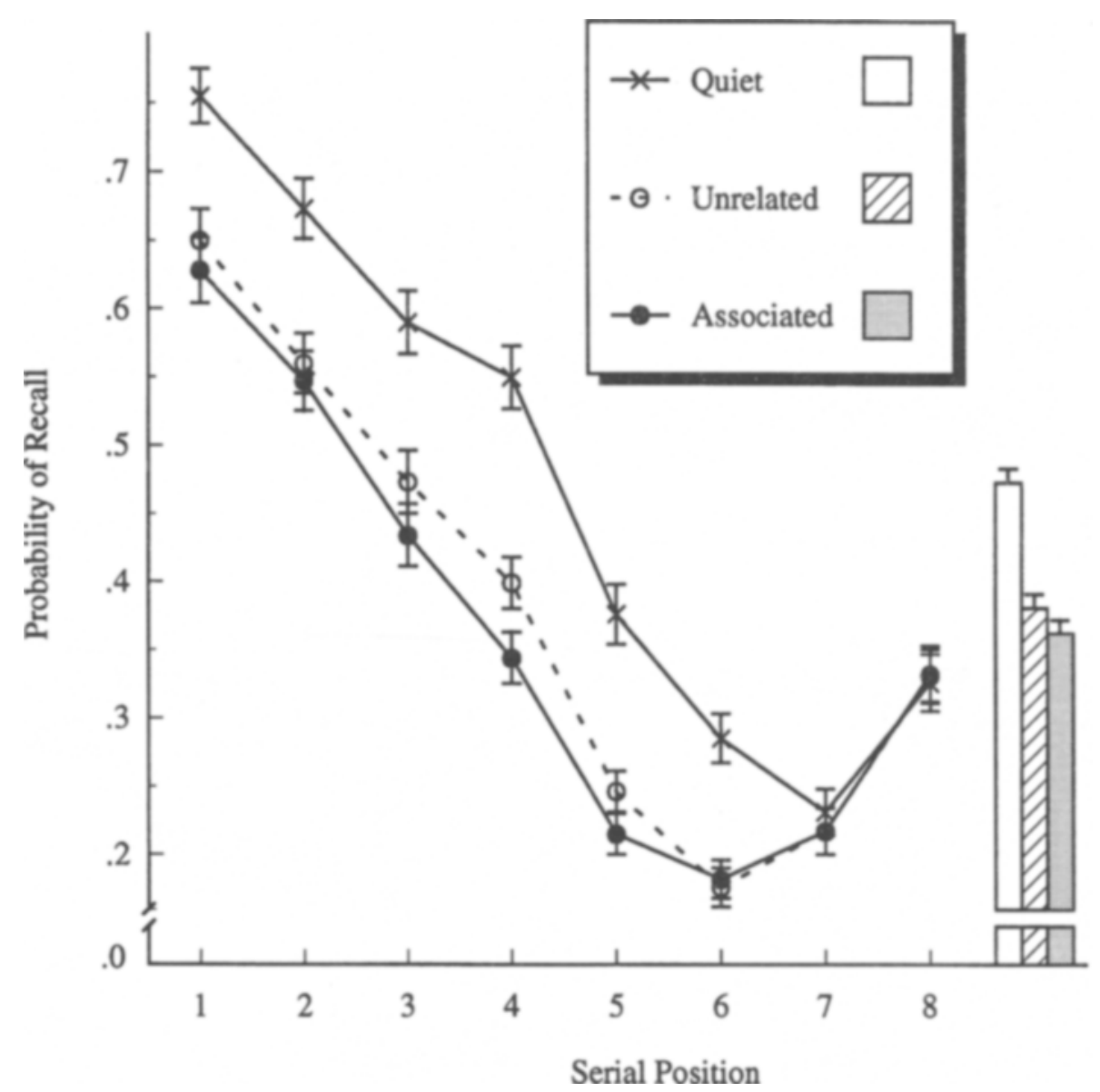

Figure 1. Mean probability of recall as a function of background condition and serial position and mean probability of recall as a function of background condition in Experiment 1. Error bars represent the standard error of the mean.

we used free recall of categorically related words (e.g., birds). LeCompte (1994; see also LeCompte et al., 1997; see Watkins \& Allender, 1987) showed that irrelevant speech disrupts free recall as well as serial recall, and we suspected that constructing a list from a semantically defined set would increase the probability that participants would use semantic processing to accomplish the task. Finally, to further increase our chances of finding an effect of semantic similarity, we used low-typicality exemplars (e.g., $e m u$ ) as the to-be-remembered visual stimuli, and we used high-typicality exemplars (e.g., robin) as the irrelevant speech.

The central purpose of Experiment 2 was to replicate the finding in Experiment 1 that semantically similar irrelevant speech disrupts recall more than irrelevant speech that is unrelated to the to-be-remembered stimuli.

\section{Method}

Participants. The participants were 60 students enrolled in psychology courses at Louisiana State University who volunteered in exchange for extra credit.

Materials and Design. We constructed 30 lists of words, each of which comprised 16 exemplars drawn from the same semantic category (e.g., fruits). These exemplars were selected from the Battig and Montague (1969) category norms. Each list was drawn from a different category. For each category, the norms are organized from the most typical to the least typical exemplars, and the 16 tobe-remembered words for each list were selected from 9th most typical to the 24th most typical exemplars for that category. The only exception was for exemplars comprising multiple words (e.g., bow and arrow). In those cases, the next most typical exemplar on the list was used as a replacement. For each list, the order of the exemplars was determined randomly and was the same for all participants. The 30 lists were randomly divided into two groups of 15 lists.

We also constructed 30 sets of irrelevant speech backgrounds. Each set consisted of the eight most typical exemplars in one of the categories used for the to-be-remembered stimuli. These 30 words were also divided into two groups of 15 lists.

There were three irrelevant speech conditions: quiet, related, and unrelated. Half of the participants saw one group of 15 lists, and the other half of the participants saw the other group of 15 lists. For all participants, the 15 lists were divided into five blocks. In each block, the three lists were each assigned randomly to one of the irrelevant speech conditions. To control for order effects, the order of irrelevant speech conditions in each three-list block were counterbalanced across participants so that all six possible orderings of the conditions were encountered by an equal number of participants.

When a list was assigned to the quiet condition, no irrelevant speech was presented. When a list was assigned to the related con- 


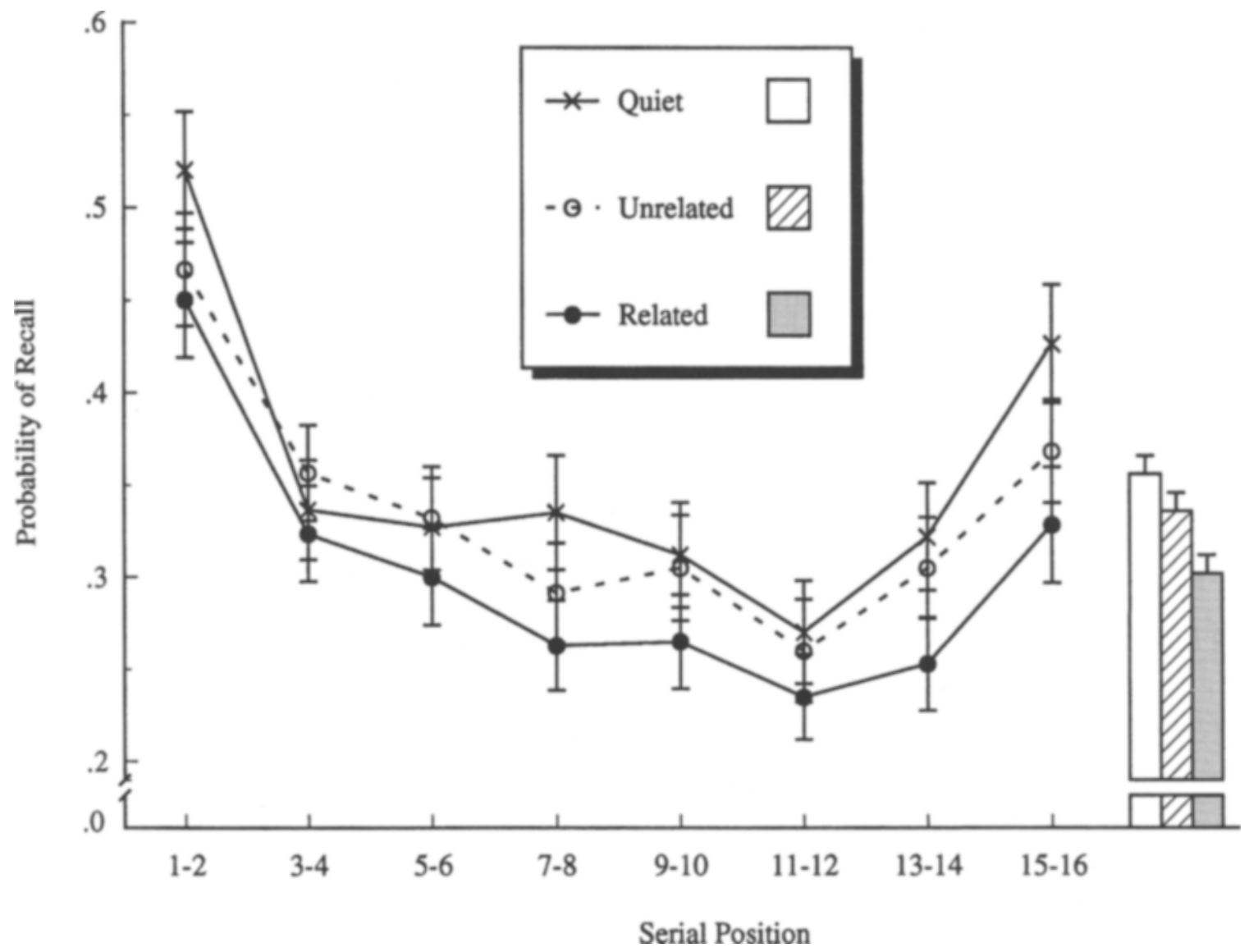

Figure 2. Mean probability of recall as a function of background condition and serial position and mean probability of recall as a function of background condition in Experiment 2. Error bars represent the standard error of the mean.

dition, the eight high-typicality exemplars for that list's category were presented as irrelevant speech. Thus, if the list comprised fruits, the irrelevant speech would also comprise fruits. When a list was assigned to the unrelated condition, a set of eight high-typicality exemplars from another category was presented as irrelevant speech. The unrelated category was always selected from the 15 categories that were not used for any to-be-remembered lists for that group of participants. Thus, if the list comprised fruits, the irrelevant speech might comprise tools, a category that was not used to generate a to-be-remembered list for that group of participants.

Each list was presented visually in the center of an Apple Macintosh Classic II computer screen. The words appeared in 48-point Geneva font in black on a white background. Each word was visible for $500 \mathrm{msec}$ and was followed immediately by the next word. All lists were presented without any accompanying irrelevant speech. The visual presentation of the words was followed by an 8$\mathrm{sec}$ quiet interval or by $8 \mathrm{sec}$ of irrelevant speech. For the two speech backgrounds, the set of eight words was presented twice at a rate of $500 \mathrm{msec}$ per word. Next, recall of the words was accomplished by writing the words in any order on an answer sheet with columns of 16 lines for each list.

Procedure. Each participant sat at a computer and wore headphones attached to that computer. The instructions were presented on the computer screen and familiarized the participants with the nature of the task. The participants saw a list of words one at a time. Following this visual presentation, participants heard the auditory presentation while the screen presented the phrase "Don't write yet." After this interval, the screen presented the phrase "Write all of the words you saw." Participants had $30 \mathrm{sec}$ to write down all of the words that they could remember in any order on an answer sheet. After the 30 -sec free recall session, a beep indicated that the participants should look at the computer screen for the next list presentation.

\section{Results and Discussion}

Figure 2 shows probability of recall as a function of background condition and serial position and as a function of background condition alone. The overall probability of recall was .36 for quiet, .34 for the unrelated background, and 30 for the related background, yielding a significant main effect of background condition $[F(2,118)=$ $\left.19.94, M S_{\mathrm{e}}=0.035, p<.001\right]$. As with the previous experiment, performance in the quiet condition exceeded performance in both the unrelated $[t(59)=2.38, p=.02]$ and the related $[t(59)=6.25, p<.001]$ speech conditions. The most important finding, however, was that the semantically related condition had a lower probability of recall than the unrelated condition $[t(59)=3.88, p<.001]$, thereby replicating Experiment 1 in demonstrating that 


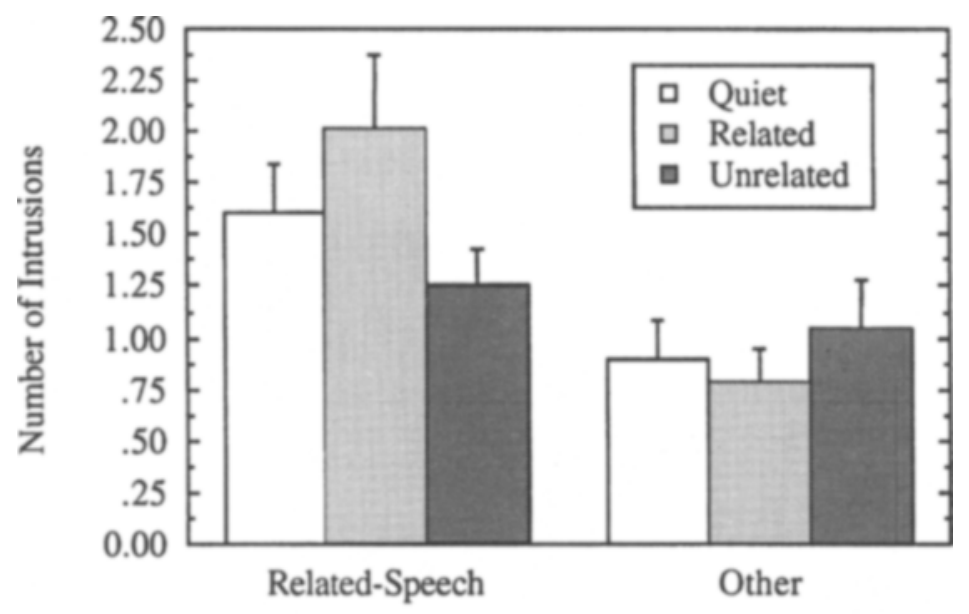

Intrusion Type

Figure 3. Number of intrusions by background condition for Experiment 2.

increasing the degree of semantic similarity between the irrelevant speech and the to-be-remembered visual items increases the magnitude of the irrelevant speech effect.

Although not of primary interest, there was also a significant effect of serial position $\left[F(15,885)=16.30, M S_{\mathrm{e}}=\right.$ $0.05, p<.001]$ resulting from the bow-shaped curve characteristics of free recall, though the recency effect was somewhat diminished, presumably because recall was delayed (see LeCompte, 1994, Experiment 2). There was no significant interaction between background condition and serial position $\left[F(30,1770)=0.59, M S_{\mathrm{e}}=0.043, p=\right.$ .96]. These data are consonant with those of LeCompte (1994), who conducted a number of experiments using free recall and found no consistent interactions between serial position and background condition (see also Jones \& Macken, 1995a; LeCompte \& Shaibe, 1997; Salamé \& Baddeley, 1982, for inconsistent interactions between background condition and serial recall).

In addition to analyzing correct recall, we examined participants' extralist intrusions. For each list, we divided intrusions into two groups: (1) those intrusions that matched one of the eight to-be-ignored words presented as irrelevant speech when that list was in the relatedspeech condition and (2) all other intrusions. The former type of intrusion, a related speech intrusion, was classified as such even when a given participant had studied that list in quiet or with unrelated speech. This classification in the quiet and unrelated speech conditions provided a base rate for falsely recalling the high-typicality exemplars used as irrelevant speech. Although we had no specific hypotheses in mind, we were interested in determining whether the irrelevant speech items showed up in participants' recall.

A 3 (background condition) $\times 2$ (type of intrusion) within-subjects analysis of variance (ANOVA) showed no main effect of background condition $[F(2,118)=1.20$, $\left.M S_{\mathrm{e}}=1.58, p=.30\right]$, but did show a significant main ef- fect of intrusion type $\left[F(1,59)=16.39, M S_{\mathrm{e}}=2.78, p<\right.$ $.001]$, in which a related speech intrusions $(M=1.62)$ were more common than other intrusions $(M=0.91)$. More importantly, there was a significant interaction between background condition and intrusion type $[F(2,118)=4.86$, $\left.M S_{\mathrm{e}}=1.65, p=.009\right]$. Figure 3 depicts this interaction.

To further explore this interaction, we conducted simple effects tests. We found no significant effect of background for the intrusions classified as "other" $[F(2,118)=$ $\left.1.65, M S_{\mathrm{e}}=0.93, p=.32\right]$. There was, however, a significant effect of background for the intrusions classified as speech related $\left[F(2,118)=3.84, M S_{\mathrm{e}}=2.30, p=.02\right]$. We compared each pair of means; however, because these comparisons were post hoc rather than a priori, we used Bonferonni's correction to reduce the possibility of Type I error: We divided our alpha of .05 equally among our three comparisons, resulting in a significance level of .017 for each comparison. Using this corrected alpha level, we found that the number of related speech intrusions for the quiet background was not reliably different from either the related $[t(59)=1.50, p=.14]$ or the unrelated $[t(59)=1.26, p=.21]$ background condition. The related and unrelated background conditions did, however, differ from each other to a significant extent $[t(59)=$ $2.77, p=.007]$.

Thus, we see that in the related speech condition, participants produced slightly more of the to-be-ignored items than they did in the unrelated speech condition. Note, however, that the rate of production of these hightypicality exemplars for the quiet condition fell between those of the other two conditions, suggesting perhaps that related irrelevant speech enhances, while unrelated irrelevant speech inhibits, false recall of category-typical exemplars. Because the absolute number of speech related intrusions was very low and quite variable, we might need considerably more statistical power to appropriately address this question. For now, the analysis of in- 
trusions suggests that one possible mechanism by which semantic similarity increases the disruptive effect of irrelevant speech is by increasing false recall, an effect that may be due to increased output interference (see Roediger, 1974) or to something akin to part-set cuing inhibition (see Watkins \& Allender, 1987).

\section{GENERAL DISCUSSION}

These experiments show that semantic similarity has a role in the irrelevant speech effect. Experiment 1 showed a small disruptive effect of semantic similarity when the visual words and the background words were strongly associated with the to-be-remembered words relative to a background of unrelated words. Experiment 2 replicated the semantic similarity effect when the task was free recall of categorically related lists.

One important conclusion that can be drawn from these findings is independent of specific theories: Whereas it was previously believed (e.g., Jones, 1993, 1995; Jones et al., 1996) that semantic meaning was unimportant to the effect of irrelevant speech in immediate recall, these experiments show that it is important. Therefore, previous attempts to argue (see, e.g., Jones, 1993, or LeCompte, 1994) that disruptive effects of irrelevant speech in tasks such as the Stroop effect (Cowan \& Barron, 1987) and reading comprehension (Martin, Wogalter, \& Forlano, 1988) are qualitatively different from the disruptive effects seen in immediate recall may be inappropriate. At least under some circumstances, all of these tasks show effects of semantic similarity. A truly comprehensive theory of the irrelevant speech effect should be broad enough to explain speech's disruptive effects wherever they might occur.

What are the theoretical implications of the present findings? First, for the phonological store hypothesis (Salamé \& Baddeley, 1982, 1989), the findings are quite problematic. The hypothesis states that the mechanism by which irrelevant speech disrupts memory is phonological similarity. There is no mechanism by which semantic similarity could affect short-term memory. One could try to propose that it is phonological similarity and not really semantic similarity that is at work in our experiments, but as mentioned earlier, the latest data on this topic (Bridges \& Jones, 1996; Jones \& Macken, 1995b; LeCompte \& Shaibe, 1997) argue strongly against any effect of phonological similarity between the to-be-remembered stimuli and the irrelevant speech. Furthermore, findings by Jones and Macken (1993) and LeCompte et al. (1997) that pure tones can produce an irrelevant "speech" effect cannot be explained by this model. Consequently, the phonological store theory no longer seems a viable explanation of the irrelevant speech effect, and thus an explanation of the present data based on phonological similarity would seem forced.

The present data also have theoretical implications for the changing state hypothesis of the irrelevant speech effect. This hypothesis, part of Jones's (1993; Jones et al.,
1996) object-oriented episodic record (O-OER) model, predicts that greater disruption will result from a greater number of changes in state. According to the model, auditory and visual stimuli form objects on a metaphorical blackboard, and if there is a change in state, an object is linked automatically for auditory stimuli and through conscious serial rehearsal for visual stimuli to the subsequent stimulus. According to Jones and Macken (1995a; see also Jones et al., 1996), objects are formed at a precategorical level - that is, before they are interpreted semantically. Once on the blackboard, objects retain no information about their original modality or meaning, and the irrelevant speech effect results from confusion among the serial order links between the stream of to-beremembered objects and the stream of irrelevant speech. In short, the O-OER theory cannot account for the semantic similarity effects in Experiments 1 and 2 because the theory explains the irrelevant speech effect as a precategorical phenomenon.

In summary, we think it is reasonable to conclude that semantic similarity is important to the irrelevant speech effect. Current theories of the irrelevant speech effect are inconsistent with this conclusion. Future theories, however, must be able to account for the effect of semantic similarity in the irrelevant speech effect.

\section{REFERENCES}

BADDELEy, A. D. (1986). Working memory. Oxford: Oxford University Press.

Battig, W. F., \& Montague, W. E. (1969). Category norms for verbal items in 56 categories: A replication and extension of the Connecticut category norms. Journal of Experimental Psychology Monographs, 80 (3, Pt. 2).

Brlodeau, E. A., \& Howell, D. C. (1965). Free association norms by discrete and continuous methods. Washington, DC: Office of Naval Research.

Bridges, A. M., \& Jones, D. M. (1996). Word dose in the disruption of serial recall by irrelevant speech: Phonological confusions or changing state? Quarterly Journal of Experimental Psychology, 49A, 919-939.

Buchner, A., Irmen, L., \& ERdfelder, E. (1996). On the irrelevance of semantic information for the irrelevant speech effect. Quarterly Journal of Experimental Psychology, 49A, 765-779.

COlle, H. A., \& Welsh, A. (1976). Acoustic masking in primary memory. Journal of Verbal Learning \& Verbal Behavior, 15, 17-32.

Cowan, N., \& BARRon, A. (1987). Cross-modal, auditory-visual Stroop interference and possible implications for speech memory. Perception \& Psychophysics, 41, 393-401.

JONES, D. M. (1993), Objects, streams, and threads of auditory attention. In A. D. Baddeley \& L. Weiskrantz (Eds.), Attention: Selection, awareness, and control (pp. 87-104). Oxford: Oxford University Press.

JONES, D. M. (1995). The fate of the unattended stimulus: Irrelevant speech and cognition. Applied Cognitive Psychology, 9, S23-S38.

Jones, D. M., Beaman, P. C., \& Macken, W. J. (1996). The objectoriented episodic record model. In S. E. Gathercole (Ed.), Models of short-term memory (pp. 209-238). London: Erlbaum.

Jones, D. M., \& MACKEN, W. J. (1993). Irrelevant tones produce an irrelevant speech effect: Implications for phonological coding in working memory. Journal of Experimental Psychology: Learning, Memory, \& Cognition, 19, 369-381.

JONES, D. M., \& MACKEN, W. J. (1995a). Organizational factors in the effect of irrelevant speech: The role of spatial location and timing. Memory \& Cognition, 23, 192-200.

JONES, D. M., \& MACKEN, W. J. (1995b). Phonological similarity in the irrelevant speech effect: Within- or between-stream similarity. Jour- 
nal of Experimental Psychology: Learning, Memory, \& Cognition, 21, 103-115.

Jones, D. M., Madden, C., \& Miles, C. (1992). Privileged access by irrelevant speech to short-term memory: The role of changing state. Quarterly Journal of Experimental Psychology, 44A, 645-669.

LECOMPTE, D. C. (1994). Extending the irrelevant speech effect beyond serial recall. Journal of Experimental Psychology: Learning, Memory, \& Cognition, 20, 1396-1408.

LECOMPTE, D. C. (1995). An irrelevant speech effect with repeated and continuous background speech. Psychonomic Bulletin \& Review, 2 , 391-397.

LECOMPTE, D. C. (1996). Irrelevant speech, serial rehearsal, and temporal distinctiveness: A new approach to the irrelevant speech effect. Journal of Experimental Psychology: Learning, Memory, \& Cognition, 22, 1154-1165.

LeCompte, D. C., Neely, C. B., \& Wilson, J. R. (1997). Irrelevant speech and irrelevant tones: The relative importance of speech to the irrelevant speech effect. Journal of Experimental Psychology: Learning, Memory, \& Cognition, 23, 472-483.

LeCompte, D. C., \& Shaibe, D. M. (1997). On the irrelevance of phonological similarity to the irrelevant speech effect. Quarterly Journal of Experimental Psychology, 50A, 100-118.

MACKEN, W. J., \& JONES, D. M. (1995). Functional characteristics of the inner voice and the inner ear: Single or double agency? Journal of Experimental Psychology: Learning, Memory, \& Cognition, 21, 436-448.
Martin, R. C., Wogalter, M. S., \& Forlano, J. G. (1988). Reading comprehension in the presence of unattended speech and music. Journal of Memory \& Language, 27, 382-398.

RoEDIGER, H. L., III (1974). Inhibiting effects of recall. Memory \& Cognition, 2, 261-269.

Salamé, P., \& Baddeley, A. D. (1982). Disruption of short-term memory by unattended speech: Implications for the structure of working memory. Journal of Verbal Learning \& Verbal Behavior, 21, 150-164.

Salamé, P., \& BadDeley, A. D. (1989). Effects of background music on phonological short-term memory. Quarterly Journal of Experimental Psychology, 41 A, 107-122.

WATKINS, M. J., \& ALLENDER, L. E. (1987). Inhibiting word generation with word presentations. Journal of Experimental Psychology: Learning, Memory, \& Cognition, 13, 564-568.

\section{NOTE}

1. In experiments on the irrelevant speech effect, the presentation of a given item as background speech and the presentation of that same item as a to-be-remembered stimulus are either uncorrelated or explicitly prevented from co-occurring.

(Manuscript received March 12, 1997; revision accepted for publication July 7,1997 .) 